\title{
BRASIL y CHINA: CAMINOS DE FORTALEZAS Y DESCONCIERTOS ${ }^{1}$
}

\begin{abstract}
Pierre Salama $^{2}$
Fecha de recepción: 02 de mayo de 2016. Fecha de aceptación: 02 de septiembre de 2016.

RESUMEN

¿El éxito económico de China ha ocasionado el fracaso económico de Brasil? o ¿debemos buscar en otro lugar las causas del desastre en el que hoy se hunde la nación sudamericana? Hay una forma para salir de la trampa rentista, pero es estrecha. Los gobiernos brasileños convencidos de que los precios crecientes de las materias primas continuarían, que sin hacer reformas estructurales podrían seguir políticas orientadas a reducir la desigualdad salarial, y disminuir la pobreza, sin tener que preocuparse por la pérdida de competitividad, dejando de lado al $1 \%$ más rico que se hace más rico, han preferido no solicitar préstamos. Las crecientes relaciones asimétricas con China han permitido la crisis, mas no el crecimiento.
\end{abstract}

Palabras clave: crisis económica, desindustrialización, inversiones, industria manufacturera, materias primas, relaciones económicas.

Clasificación JEL: E22, E32, F13, O13, O14.

\section{Brazil and China: Paths of Strengths and Turmoil}

\begin{abstract}
Has China’s economic success brought about Brazil's economic failure? Or should we look elsewhere for the causes behind the disaster into which the South American nation is currently sinking? There is a path out of the rentier trap, but it is narrow. Brazilian administrations have preferred not to pursue loans, convinced that raw materials prices would keep rising, and that even without making structural changes, it would be possible to enact policies designed to reduce the wage gap and mitigate poverty, with no concern for declining competitiveness, and a blind eye to the top $1 \%$, which is becoming wealthier. An increasingly asymmetrical relationship with China has made room for crisis, but not growth.
\end{abstract}

Key Words: Economic crisis, deindustrialization, investment, manufacturing industry, raw materials, economic relations.

1 Traducción realizada por Marcia Luz Solorza, profesora de la Facultad de Economía, Universidad Nacional Autónoma de México, UNAM.

2 Universidad de París XIII, Francia. Correo electrónico: psalama@wanadoo.fr 


\section{BRÉSIL ET CHINE: ROUTES FORCES ET EMBARRAS \\ Résumé}

Est-ce que les succês économique de la Chine a conduit â l'échec économique du Brésil?, ou de catastrophe où se coule aujoud'hui la nation sud-américaine? Il y a un moyen de sorti gouvernements brésiliens, convaincus que la hausse des prix des matières premières continuerait, et que sans réformes structurelles il pourrait poursuivre des politiques visant à réduire l'inégalité des salaires, et aussi qu'il pourrait réduire la pauvreté sans tenir compte de la perte de compétitivité. En mettant de côté au $1 \%$ plus riche que deviennent plus riches, ils ont choisi ne pas demander des prêts. L'accroissement des relations asymétriques avec la Chine ont conduit â la crise, mais pas la croissance.

Mots clés: crise économique, la désindustrialisation, les investissements, secteur manufacturier, les matiêres premiêres, le relations économiques.

\section{BRASIL E CHINA: CAMINHOS DE FORTALEZAS E EMBARAÇOS \\ Resumo}

O sucesso económico da China levou ao fracasso econômico do Brasil? Ou devemos procurar noutro lugar as causas do desastre pelo qual a nação sul-americana afunda hoje? Existe uma maneira de sair da armadilha rentista, mas é difícil. Os governos brasileiros estavam convencidos de que o aumento dos preços das matérias-primas continuaria, que sem fazer reformas estruturais poderiam prosseguir as políticas destinadas a reduzir a desigualdade salarial e reduzir a pobreza, sem ter que se preocupar com a perda de competitividade, deixando de lado $1 \%$ mais rico que fica mais rico, preferiram não solicitar empréstimos. As crescentes relações assimétricas com a China levaram à crise, mas não ao crescimento.

Palavras-chave: crise económica, desindustrialização, investimentos, indústria manufatureira, matérias-primas, relaçóes económicas.

\section{巴西和中国：巩固与迷茫之路}

皮埃尔・萨拉玛

摘要

中国经济的腾飞导致了巴西经济的失败? 还是我们应该在其他地方寻找今 日巴西所陷经济灾难的原因? 中等收入陷阱是有一条出路的, 但是出路是 很狭窄的。巴西政府深信原材料价格将继续上涨, 深信他们不需要进行结 构性改革仍然可以继续施行降低工资不平等的政策，减少贫困。他们对只 占人口 $1 \%$ 的富人坐视不管, 选择不申请贷款, 也并不担心因此而失去竞争 力。而巴西与中国日益增长的不对称关系加深了巴西的经济危机, 对经济 增长并没有帮助。

关键词: 经济危机, 去工业化, 投资, 制造业, 原材料, 经济关系 


\section{INTRODUCCIÓN}

El aumento del precio de las materias primas y de los volúmenes de exportación han modificado las presiones externas de Brasil en los años 2000, en la actualidad pesan menos que antes. Este nuevo contexto es conocido como "viento de cola". Éste favoreció: 1) un crecimiento mayor que en la década de los noventa; 2) una acentuada desindustrialización; 3) una inserción en la división internacional del trabajo sustentada en productos con menor uso intensivo de tecnología (a excepción de unos pocos sectores como la aeronáutica), conduciendo, esta falta de generación de productos industriales, desde el año 2008 a un déficit de la balanza comercial, lo cual rápidamente se ha convertido en un abismo, sin alcanzar poco a poco a subsanar el excedente proveniente de la venta de materias primas; 4) en resumen, el desfase entre la demanda y la oferta de mano de obra en la industria ocasionó una disminución de la demanda de mano de obra calificada por parte de las empresas, incluso, relativamente por debajo de la oferta, la cual había venido aumentado gracias a los esfuerzos realizados en materia de educación (incremento del número de ańos escolares).

El "viento de cola" permitió a la vez un aumento del salario mínimo y de los salarios en general, y un aumento significativo de las políticas sociales. Pero también favoreció un comportamiento más o menos oportunista por parte de los gobiernos, mismo que ha generado la profunda crisis económica que se vive actualmente. La ralentización económica, posterior a la crisis, se inscribe en el régimen de crecimiento centrado en la reprimarización de la economía y la entrada de capitales. Es por ello que la caída de los precios de las materias primas, no ha hecho más que precipitar una crisis latente. El oportunismo de los gobiernos ha sido creer "que lo que había funcionado", podría durar y que no era necesario prepararse para enfrentar futuros conflictos de clases, la abundancia de divisas podía permitir eludirlos.

Así que China no es la causa de que Brasil esté en crisis. La relación entre Brasil y China recuerda una canción de un cantautor francés, Serge Gainsbourg, que dice: "Te amo, yo ya no más"; "te amo", porque esa relación ha aportado la riqueza, "ya no más", porque esa riqueza ha aportado el germen de la crisis que no han sabido o no han querido evitar los gobiernos aplicando políticas apropiadas.

China y Brasil, dos países con condiciones económicas diferentes y de relaciones estrechas, pero asimétricas, componen una dupla en la que uno tiene un rumbo económico donde la reconversión es el alfa y omega de su crecimiento, y el otro aprovecha las demandas que le hace China, pareciendo un 
regreso al pasado por su re-especialización en las actividades mineras. El éxito (económico) de uno hace la desgracia (económica) del otro, o bien ¿debemos buscar en otra parte las causas del desastre en que se hunde hoy en día Brasil?

\section{ALGUNOS HECHOS ESTILIZADOS: LO QUE DIFERENCIA A BRASIL Y CHINA}

\section{1) El PIB per cápita}

En los años ochenta, América Latina estaba atrapada y fue superada por los dragones asiáticos (Corea del Sur, Taiwán, Hong-Kong y Singapur). Sin embargo, en el periodo 1990-2000, América Latina vuelve a moderar el crecimiento y el ingreso per cápita de los principales países de la región (Argentina, Brasil, México, Colombia...), en 2012 ascendía a poco más de una cuarta parte de los ingresos de Estados Unidos, mientras que los ingresos per cápita de Corea del Sur y Taiwán se situaban alrededor del 70 y $80 \%$ de los ingresos de Estados Unidos. A pesar de ese crecimiento más elevado, América Latina se marginaliza. En 1980, en China el ingreso per cápita ascendió a 306.7 dólares medidos en poder adquisitivo (PPA) y en Brasil a 4809.6 dólares PPA. En el año 2015, es muy cercana 14107.4 dólares PPA y 15614 dólares PPA, respectivamente (FMI). Entre 1980 y 2015 el Producto Interno Bruto (PIB) per cápita se multiplicó por 46 en China, mientras que en Brasil sólo lo hizo por 3.25.

El curso económico en ambas naciones es muy diferente. En China el crecimiento es impulsado por un impresionante aumento de las exportaciones de productos manufacturados y un exceso de inversión; en Brasil, en los años 2000, el motor son las exportaciones de materias primas, y el crecimiento es frenado por la falta de inversión. El mayor crecimiento en uno (China) impulsa un débil crecimiento global en el otro (Brasil), ligeramente superior al alcanzado en los años noventa.

A la llegada del presidente Lula da Silva al poder (2003), el crecimiento se aceleró ligeramente, disminuyó la pobreza de manera drástica, pero al contrario de lo que oficialmente se dice, los ricos se hacen más ricos, y las ganancias de este $1 \%$ de la población crecen al igual que en los países desarrollados. Lo que Brasil está haciendo no es una excepción, salvo su capacidad para disminuir la pobreza. Muchos economistas cuestionan la posibilidad de que Brasil se convierta en un nuevo "El Dorado". Sin embargo, el mito se derrumbó desde 2011 y los sueños vuelan, el milagro se hace espejismo, incluso pesadilla a partir de 2014. 


\section{2) Demografía}

China (1 400 millones de habitantes) está densamente poblada en comparación con Brasil (207 millones de habitantes). Cuando se toman en cuenta las desigualdades de ingresos y su evolución, el impacto de la demografía sobre el tamaño de los mercados internos es diferente en los dos países: a) la desigualdad de ingresos en China es similar a la observada en Brasil. Desde ese punto de vista China ha sido latino-americanizada, la creciente producción de desigualdades es cada vez más importante. Sin embargo, esto último no constituye un obstáculo para el crecimiento continuo debido, precisamente, a la importancia de bienes de consumo, bienes intermedios, bienes no duraderos y bienes de capital vinculados al aumento poblacional. En China, el aumento de la capacidad ociosa en la producción, más que a la insuficiencia de la demanda y a la especulación inmobiliaria, se debe más a las decisiones políticas tomadas a nivel provincial porque han permitido la proliferación de fábricas dedicadas a la producción de un mismo producto en cada una de las provincias.

No obstante, ese importante problema, el auge de la producción industrial verifica la Ley de Kaldor: "más industria, más productividad" y economías de escala, y un mayor crecimiento. A pesar de que Brasil cuenta con una gran población (207 millones de habitantes), en comparación con otros países de América Latina, dicha ley no es totalmente comprobable. Las desigualdades de ingresos son muy importantes y la dimensión del mercado interno puede ser insuficiente respecto a la oferta necesaria para beneficiarse de las economías de escala, para la fabricación de determinados productos, excepto para complementar la demanda interna a través de los mercados externos como se hizo en Corea del Sur, por ejemplo.

La competencia internacional, estimulada por la liberalización del comercio, la apreciación de la moneda, la reducción de los subsidios, la cada vez más difícil producción doméstica en condiciones adecuadas de rentabilidad, el aumento de la demanda de diferentes categorías sociales, la de productos intermedios y de bienes de equipos eran, entonces, cada vez más satisfechas por un aumento de las importaciones permitidas por la desaparición de las restricciones externas, tras el aumento de las exportaciones de productos primarios y la abundancia de divisas debido al crecimiento de la inversión en el país.

El crecimiento en los años 2000 es alimentado por las actividades rentistas, la desindustrialización se afirma y la productividad evoluciona modestamente. b) La tasa de crecimiento demográfico, según el Instituto Nacional de Estadísticas y Estudios Económicos (INSEE, por sus siglas en francés) en 2011 fue del orden de $0.48 \%$ en China, a pesar de la política de un solo hijo, y actualmente 
en Brasil es de 1.1\%. Las tasas de natalidad son diferentes. En China, la política de un solo hijo, aplicada desde 1979 para frenar la explosión demográfica, condujo a un envejecimiento de la población y a una transformación de la pirámide de edades, convirtiéndose progresivamente en un obstáculo a la reanudación del crecimiento, la relación eficacia/ineficacia cada vez es más débil, de ahí el abandono de esa política en 2015.

Brasil está experimentando una transición demográfica bastante rápida: según los datos del Instituto Brasileño de Geografía y Estadística (IBGE), la tasa de natalidad en 1980 fue de $4.10,2.80 \%$ en 2000 y $2.40 \%$ en 2015 , respecto a la tasa de $1.70 \%$ observada en Europa. A medida que la transición es más rápida, la población en edad de trabajar ( 15 a 64 años) es más importante en términos relativos, son 140.9 millones de personas respecto a un total de 207 millones de habitantes. Brasil debe ofrecer empleo a esa población, lo cual implica tener que crecer a una tasa importante, sobre todo, porque el país tiene una tasa de urbanización más alta que los países industrializados. Para una tasa de crecimiento débil, el riesgo de un aumento del desempleo es elevado, incluso con una tasa baja de crecimiento de la productividad. La crisis inició en 2014, y se manifestó en 2015-2016 por una duplicación de la tasa de desempleo que ha afectado particularmente a los jóvenes y graduados. Por el contrario, en China, la migración del campo a las ciudades se ha ralentizado, y la falta de mano de obra se hace sentir, aunque la tasa de crecimiento permanece elevada.

\section{3) Los recursos naturales}

Aparte de la "tierra rara", el carbón, China no tiene materias primas para cubrir sus altas necesidades. Se ve obligada a importarlas para abastecer su crecimiento y cumplir con los nuevos requisitos relacionados con los cambios en los patrones de consumo, consecuencia propia del aumento del ingreso per cápita. Por el contrario, Brasil es rico en materias primas de origen mineral o agrícola. Esta situación se explica a la vez por el crecimiento de la producción de materias primas para la exportación y el retorno en el siglo XXI a la especialización internacional tendiente a la primarización de la economía, utilizando técnicas ultra sofisticadas que podrían ser designadas con el término "consenso de las commodities" (M. Svampa). En resumen, a fin de garantizar el suministro de materias primas, la inversión directa de China en Brasil se ha orientado a la compra de tierras o minas. China se ha convertido en el mayor socio comercial de Brasil, en 2013 el 19\% de sus exportaciones se dirigieron a él, estuvieron por delante de Estados Unidos (10\%). Igualmente, este es el caso de Chile (25\%), de Uruguay (22\%) y de Perú (18\% a la par de Estados 
Unidos). Este no es el caso de Colombia, Estados Unidos sigue siendo el socio principal (32\%), seguido de China (9\%) (FMI-DOTs). Esto se traduce en una vulnerabilidad más o menos fuerte, según el grado de apertura y la participación de las exportaciones a China en el total. Como podemos ver, en el caso de Brasil (BR) es alta, pero menor que en Chile (CL) donde el grado de apertura es más elevado y la participación de las exportaciones es más alta, o todavía más en Venezuela (VE), Perú (PE) y Uruguay (UY).

\section{LAS RELACIONES ECONÓMICAS MÁS ESTRECHAS, MARCADAS POR UNA DOBLE ASIMETRÍA}

China se ha convertido en el primer cliente de Brasil. Las relaciones entre los dos países se han estrechado a partir de los años 2000; las exportaciones brasileñas a China alcanzaron cerca de mil millones de dólares en el año 2000, y en el año 2013 fueron de 40 mil millones de dólares. En el año 2000, China era el $12^{\circ}$ socio de Brasil para las exportaciones, y el $11^{\circ}$ para las importaciones; en 2009 era su primer socio para las exportaciones, y el primero para las importaciones en 2012. Ya en 2014, las exportaciones de Brasil a China representan el $18 \%$ de sus exportaciones globales, y sus importaciones de China el 16\% (OCDE/CAF/CEPAL, 2016). La balanza comercial entre ambos países tuvo un saldo negativo en el año 2000 (137 millones de dólares), posteriormente fue positivo, excepto en 2008, a medida que han progresado las ventas de materias primas de Brasil a China. En 2011 el saldo positivo fue de 2 mil millones de dólares y enseguida declinó fuertemente. El valor de las exportaciones brasileñas a China cayó, y a su vez las importaciones retrocedieron, aunque menos que sus exportaciones, para fijarse en 3.27 mil millones de dólares en 2014 y a 4.88 mil millones de dólares en 2015 (CEPII).

Las relaciones comerciales entre China y Brasil se caracterizan por una doble asimetría: China vende bienes manufacturados a Brasil y compra materias primas. Sí China es el socio más importante de Brasil, pero este no es el caso de Brasil. Estas asimetrías tienen consecuencias en las negociaciones entre ambas naciones.

La estructura de las exportaciones de Brasil a China está dominada por la venta de materias primas y productos intensivos en recursos naturales, en 2000 significaron respectivamente el 66.7 y $13.8 \%$, y en 2011 el 83.7 y $10.3 \%$. Por el contrario, la estructura de las exportaciones de China a Brasil está dominada por la venta de bienes manufacturados, las ventas de productos primarios y bienes intensivos en recursos naturales no representaron más que 
el 8 y $13.6 \%$ respectivamente en el año 2000 , y 2.5 y $9.6 \%$ respectivamente en el año 2011, mientras que las exportaciones de bienes manufacturados intensivos en desarrollos de investigación representaron el 20.4\% (Secex/MDIC).

En el año 2013, Brasil proveyó a China 75\% de sus importaciones agrícolas procedentes de América Latina, y Argentina 16\%. Las exportaciones de soja a China representan a su vez el $37 \%$ del total de las exportaciones a ese país. Las exportaciones de productos agrícolas están fuertemente concentradas en la soja: en el año 2011, el 78\% del total de las exportaciones correspondió a la venta de soja y la harina, y 6\% al aceite de soja (OCDE/CAF/CEPAL, 2014 y 2016). La venta de soja ascendió a 15\% del total de las exportaciones de Brasil el 1 de septiembre de 2014 . $^{3}$ Previamente, sobre todo entre 2002 y 2011, las ventas aumentaron de 896 a 9513 millones de dólares para la soja y en el caso del aceite de soja pasaron de 127 a 660 millones de dólares. Las exportaciones agrícolas están altamente concentradas, por ello Brasil es particularmente vulnerable a los cambios de esta materia prima. A pesar de que el volumen de ventas de soja por parte de América Latina ha crecido un $28 \%$ en el año 2015, su valor se redujo un 7\% (China-Latin American Economic Bulletin, 2016: 3), el aumento significativo de los volúmenes no compensó la caída de los precios. Entre 2013 y 2015, el precio del grano de soja pasó de 538 a 391 dólares por tonelada métrica (véase gráfica 1).

Brasil, también exporta otras materias primas, incluyendo el mineral de hierro que continúa siendo la más importante. El valor de las exportaciones del mineral de hierro y derivados ascendió a un 35\% de sus exportaciones a China en 2014. La caída en el precio del mineral de hierro ha sido impresionante. Con un índice de 100 en 2010, su precio llegó a un índice de 28 en diciembre de 2015 (Banco Mundial, 2016), y no es sino hasta finales de 2015 que la curva se ve un poco al revés. Aunque, el volumen de mineral de hierro aumentó en un 10\% entre 2014 y 2015, la caída en el valor de las exportaciones fue especialmente alta, de 34\% (China-Latin American ...op. cit). ${ }^{4}$ (véase gráfica 2).

La producción de soja ha aumentado muy rápidamente en Brasil, en 1980-1981 la producción ascendió a 15.2 millones de toneladas métricas, y en 2014-2015 alcanzó los 100 millones de toneladas métricas, la mitad de las cuales se exportó, principalmente a China que en esa fecha importó 78.4 millones de toneladas métricas (véase Commodity Market Outlook, Quaterly Report, World Bank, Jan., 2016).

4

El panorama es bastante pesimista. Ciertamente, el consumo chino de mineral de hierro es considerable: 43.9\% del consumo promedio mundial entre 2010 y 2014 (entre 1990 y 1994 la media fue de sólo $6.4 \%$ ), pero disminuye drásticamente en los últimos años debido al exceso de oferta en el sector siderúrgico. 
Gráfica 1. Precio del grano de soja por tonelada métrica, 2005-2015

(Dólares)

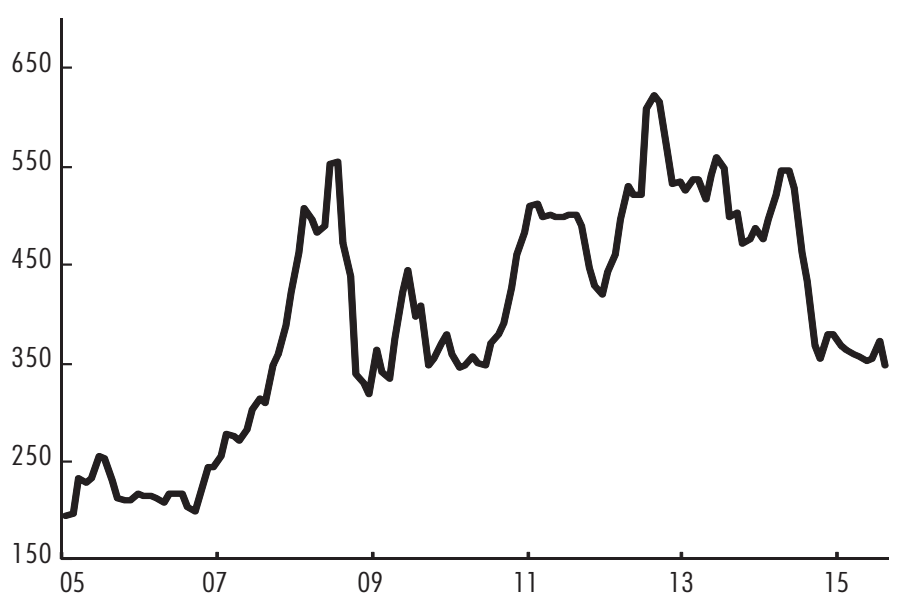

Fuente: Commodity Market Monthly, IMF, sept 2015.

Gráfica 2. Precio del mineral de hierro por tonelada métrica, 2005-2015

(Dólares)

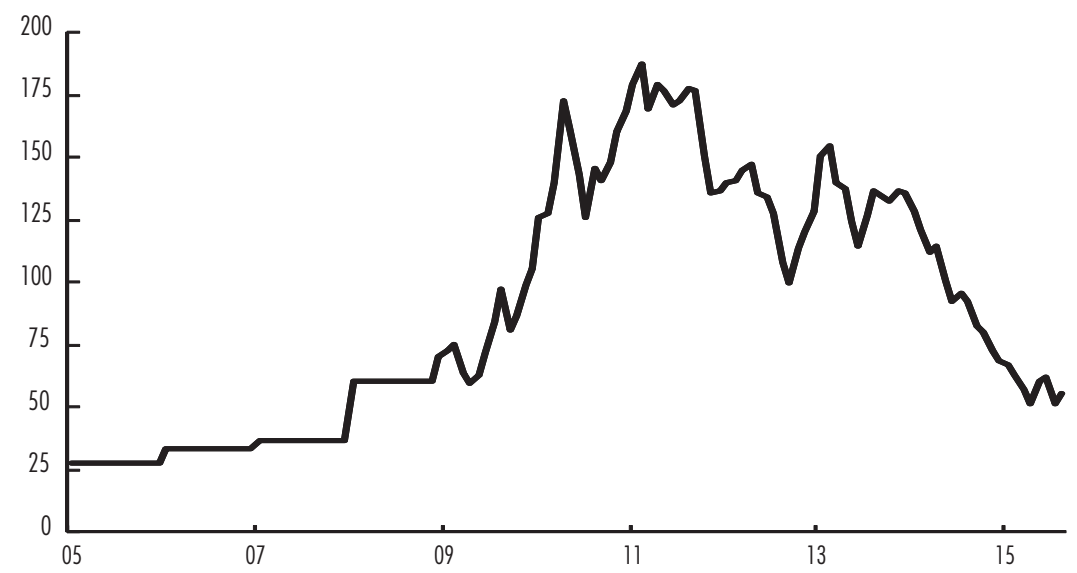

Fuente: Commodity Market Monthly, IMF, sept 2015. 
Como se observa en la siguiente gráfica, en la importancia del comercio sostenido entre Brasil y China radica la vulnerabilidad del primero, misma que aparentemente, es menor a la de Perú, Chile y Uruguay, gracias a que no es un país tan abierto comercialmente, y las posibilidades de contagio no resultan importantes.

Gráfica 3

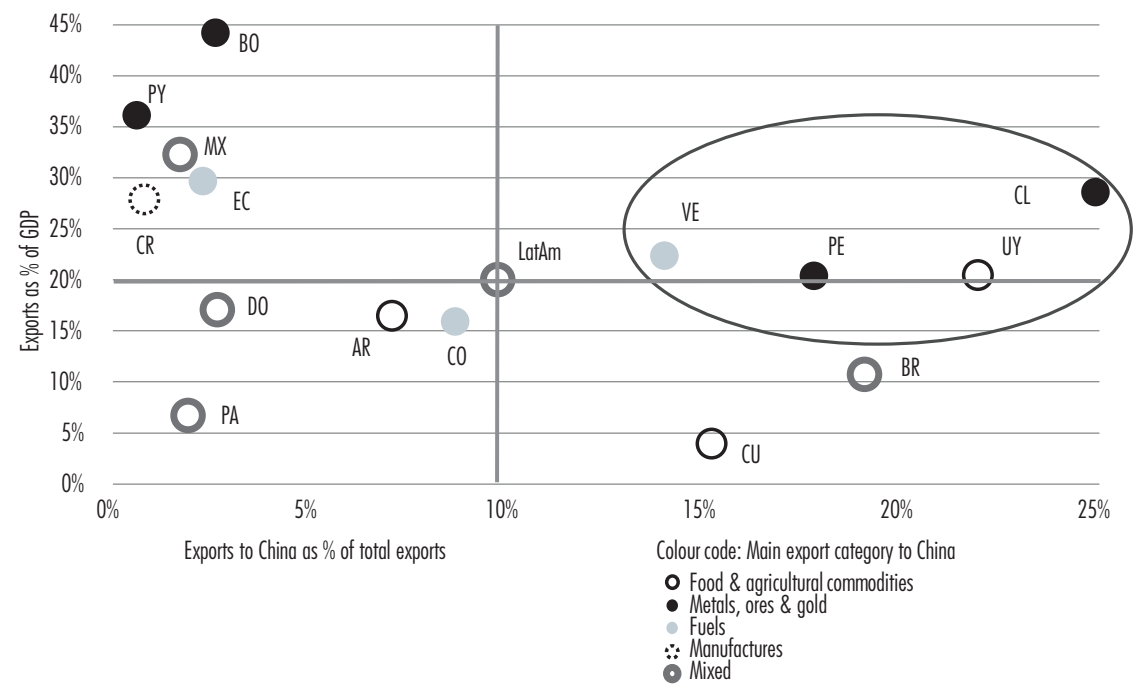

Fuente: IMF, DOTS, IMF WEO, UNCTAD, Deutsche Bank Research.

Sin embargo, debemos tener cuidado con un enfoque que se limita al análisis de estas dos variables.

Más allá del grado de apertura y la importancia relativa del comercio con China, debemos tener en cuenta el impacto del comercio en el tejido industrial, la naturaleza de los productos exportados, y cómo participan en la cadena internacional de valor. Por ejemplo, México por falta de materias primas exporta muy poco a China, en cambio importa de ella muchísimos productos de la industria de transformación. Por un dólar que México exporta a China, importa el equivalente a 10 dólares. Las relaciones comerciales que México sostiene con China, para ser más precisos, la manera en que se practica su comercio, influyen en la desindustrialización de algunos de sus sectores productivos. 
Cuadro 1. México y Brasil: evolución del índice de "amenaza competitiva" 2000, 2005, 2010 y 2013 (Porcentajes)

\begin{tabular}{lllll} 
& 2000 & 2005 & 2010 & 2013 \\
\hline México & 47.590 & 44.812 & 46.168 & 41.942 \\
Brasil & 26.174 & 21.760 & 12.726 & 14.197 \\
\hline
\end{tabular}

Fuente: OCDE/CAF/Cepal, 2016: 110.

El índice se refiere a la obra de Lall, repetido por Gallagher, el cual se calcula como el porcentaje de sectores productivos (cuatro dígitos) en los cuales, China tiene un crecimiento superior al crecimiento de las exportaciones de cualquier país en el periodo 2000-2012.

Brasil y todos los países de América Latina participan (menos que China) en la cadena de valor internacional. Se distinguen dos tipos de participación en dicha cadena: uno por detrás y otro desde el frente. El primero busca medir los bienes importados que son incorporados a las exportaciones de un país, en porcentaje de sus exportaciones brutas, sea China o Brasil. En el segundo se busca medir los bienes exportados por un país, incorporados a las exportaciones de este último en porcentaje de sus exportaciones brutas. En ambos tipos de participación, Brasil está en desventaja respecto a China. La participación de Brasil, medida por el tipo por detrás fue del $11.4 \%$ en el año 2000 y $10.7 \%$ en 2011 , mientras que en China, para los mismos años, fue de 37.2 y $32.1 \%$, respectivamente. La participación un poco más débil en el año 2011 que en 2000 y es reveladora del esfuerzo de China para integrar más sus líneas de producción. La participación en la cadena de valor por el tipo por delante para Brasil es más importante que la de China: $17.1 \%$ en 2000 y $24.5 \%$ en 2011, contra 10.8 y 15.6\% para China en los mismos ańos (oCDE/cepal/ CAF, 2015). Con la reprimarización de su economía, Brasil exporta cada vez más bienes, materias primas, a otros países, pero es China quien las incorpora en la producción de sus exportaciones. A partir de estos datos, deducimos que la fragmentación es menor en Brasil que en China, teniendo menos productos con alta elasticidad de la demanda con respecto al ingreso, por tanto, está menos sujeto a la inserción dinámica en la división internacional del trabajo que China y la mayoría de las economías asiáticas.

Avanzando aún más, la complementariedad de Brasil con China no es del orden de "ganar-ganar". Uno pierde tanto porque su especialización se centra en los cultivos comerciales y se produce con inversiones a un tiempo más o 
menos largo, por tanto, recuperarlas toma tiempo, y ello a su vez debilita a su aparato industrial. El otro gana porque obtiene materias primas que le faltan, ello favorece la flexibilidad de su aparato industrial y su voluntad de integración, y facilita el abandono de la producción de bienes que necesitan poco capital y mucha mano de obra no calificada, para dirigirse a cubrir la producción de bienes más sofisticados y más demandados, al igual que lo hicieron en su tiempo Corea del Sur y Taiwán.

La insuficiencia de materias primas, sin incluir al carbón y la escasez de tierras, tornan más vulnerable a China. Por tanto, no asombra que desde hace algunos años utilice una parte de sus reservas internacionales para multiplicar las inversiones directas en los países ricos en materias primas, favorecer el desarrollo de las infraestructuras portuarias y ferrocarrileras, y que invierta en la compra de tierras con el fin de proteger su medio ambiente. Es muy difícil tener una idea exacta de la importancia de las inversiones extranjeras directas (IED) de China en América Latina. Si tomamos las estadísticas oficiales, América Latina no es el principal destino: en 2010, América Latina recibió el 15.3\% de las inversiones chinas, en comparación con el 65\% de los países asiáticos; Brasil fue un destino marginal ( $0.7 \%$ de la IED china, fuente: Mofcon China).

Sin embargo, estos datos son infravalorados: una parte muy grande de las inversiones pasa por Hong Kong, Luxemburgo, Islas Caimán, Islas Vírgenes y no necesariamente son contabilizadas por Mofcon of China, así fue realizada la compra de $40 \%$ de Repsol-Brasil por China, la cual apareció como una compra luxemburguesa. Más globalmente, los datos de ONECH (2014) revelan que entre 2010 y 2013 las Islas Vírgenes recibieron el 51\% de las inversiones extranjeras directas chinas destinadas a América Latina, las Islas Caimán 32\%, y Brasil sólo el 3\%. Si como lo hizo Cepal en 2015, estos datos se "corrigieran", observaríamos que entre 2010 y 2015, en realidad, Brasil recibió el 56\% de la IED china, Perú 16\%, Argentina 15\%, Venezuela y Colombia 5\%, cada una.

Desde el año 2010, estas inversiones, así como los préstamos, conocieron un cierto auge sin que con precisión se pueda evaluar su importancia. Las promesas de préstamos aumentaron, igualmente sus realizaciones, sin que se conozca exactamente el escalonamiento de su tránsito de las unas a las otras. En ocasiones estos préstamos son reembolsados en materias primas, como es el caso de Venezuela. De 2013 a 2014 los préstamos fueron aumentados, en 2014 alcanzaron 22 mil millones de dólares (71\%), de los cuales a Brasil se

Véase E. Dussel Peters y S. Ortiz Velásquez (coords.). Monitor de la ofdi de China en México, núm.1, marzo, 2016. 
destinaron 8.6, a Argentina 7, y 5.7 a Venezuela, según China Latin America Finance Database Report. Las inversiones directas aumentaron por dos razones: la depreciación de las monedas latinoamericanas reduce los costos de la inversión, y las negociaciones de China y los Estados latinoamericanos, especialmente Brasil, tuvieron como resultado promesas de inversiones directas, particularmente en el ramo industrial, para compensar el aumento de las importaciones provenientes de China. Según los cálculos realizados por Financial Times y el Bureau Van Dikj (China-Latin American Bulletin, 2016), las IED chinas, por más de 10 mil millones de dólares, tuvieron como destino América Latina, en terrenos no urbanizados serían 31.3 mil millones de dólares, y las fusiones-absorciones de 18.8 mil millones de dólares entre los años 2011 y 2015. La composición de estos dos tipos de inversión no es la misma. Las inversiones en terrenos no urbanizados conciernen principalmente a los sectores: manufacturero (20\%), agricultura (18\%), logística (15\%), comunicaciones $(14 \%)$, finanzas y bienes inmuebles (14\%), adquisiciones -prioritariamente petroleras- (39\%), y sector extractivo (37\%), estos dos primeros destinos representan más de tres cuartas partes del total de las adquisiciones, el sector manufacturero sólo significa el 1\%.

La estrategia de China es diferente a la desarrollada frente a los países avanzados. La compra de empresas extranjeras no tiene como objetivo la adquisición de tecnologías extranjeras sino, principalmente, asegurarse el abastecimiento de materias primas. En estos dos sectores, las fusiones o absorciones son proporcionalmente más importantes que las operadas en el resto del mundo, al ascender a 15\%. A la inversa, las fusiones-absorciones procedentes de China, en el sector manufacturero, casi-inexistentes, se elevan al $13 \%$ para el resto del mundo. Las inversiones directas de China destinadas al sector manufacturero, principalmente se dirigen a Brasil y en menor medida a Argentina.

\section{¿CHINA ES RESPONSABLE DEL DÉBIL CRECIMIENTO DE BRASIL Y DE SU DESINDUSTRIALIZACIÓN?}

Es nula la necesidad de referirse a las etapas de desarrollo teorizadas en su tiempo por Rostow para reconocer que hay trayectos portadores de futuro, y otros que no. Podemos reagrupar a las economías en cuatro categorías: 1) economías atrasadas (emergentes o no), 2) "tigres", 3) "campeones", y 4) economías maduras, a partir de una gráfica en cuatro cuadrantes ordenando la densidad industrial medida en valor agregado por habitante en dólares 
en el eje de las ordenadas, y en el eje de las abscisas peso del sector manufacturero respecto al PIB, el cruzamiento de las ordenadas y las abscisas se sitúa en 3 mil dólares y el 18\%.

Las principales economías emergentes se ubican en el primer cuadrante. Su densidad industrial no es muy importante y el peso de sus manufacturas en el PIв es igualmente débil, este no es el caso de las "tigres" cuya densidad es sin duda relativamente más o menos débil, pero su peso es más importante. Su éxito, en términos de crecimiento, baja volatilidad y la durabilidad de ésta, sugiere aumentar la importancia relativa de la industria. La etapa más alta es consecuencia lógica de ese crecimiento más elevado, pero permite se manifiesten los efectos de una desindustrialización relativa y el peso de los servicios se vuelve cada vez más importante. La etapa final, la de las economías maduras, se caracteriza por una tendencia al estancamiento secular. Ella es especialmente para las economías avanzadas (véase gráfica 4).

Este posible trayecto económico - de cuadrante a cuadrante- sugiere que esta gráfica es una aplicación de la Ley de Kaldor, que mencionamos anteriormente. A partir de 1935 y 1980, las economías de América Latina se mueven de izquierda a derecha y de abajo (en la media) hacia arriba en el primer cuadrante. No pudieron pasar al segundo cuadrante como lo hicieron en su tiempo Corea del Sur o bien Taiwán. Por el contrario, con la reprimarizacion, se desplazaron de la derecha hacia la izquierda en el seno del primer cuadrante. Entonces, ¿hay que vincular el modesto crecimiento, relativamente volátil y poco sostenible de las principales economías latinoamericanas a su incapacidad de aumentar el peso de sus manufacturas en el PIB? ¿Podemos explicar la desindustrialización de Brasil por su reprimarización y por sus cada vez más privilegiadas relaciones comerciales con China? En fin, entonces, ¿podemos atribuir a China la responsabilidad de este crecimiento débil, y hoy de la crisis que, sería provocada por la caída de los precios de las materias primas?

Brasil, así como otros tantos países de América Latina, escapó de la recesión externa gracias a la entrada de capitales del extranjero, y gracias a la subida de los precios de las materias primas y al fuerte aumento de los volúmenes exportados. Esta doble subida ha sido alimentada por el fuerte, largo y excepcional crecimiento de China. La abundancia de divisas puso en marcha un proceso de apreciación de la moneda nacional en el periodo 2004-2011, sin que los sucesivos gobiernos hubieran procurado disminuirlo, salvo de manera errática en el periodo 2011-2014, en el momento de la primera presidencia de Dilma Roussef al frente de Brasil (véase gráfica 5).

Según Nassif et al. (2015), con la finalidad de facilitar la lectura de la gráfica, recordemos que una curva decreciente significa una apreciación de 
Gráfica 4. Configuración para el año 2013

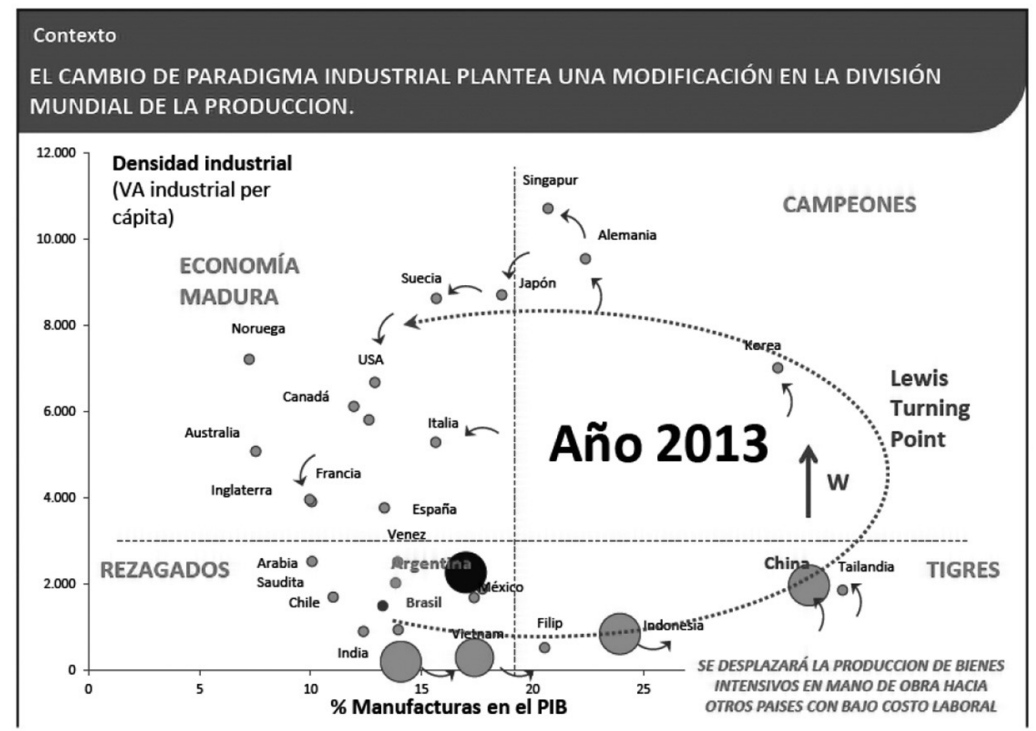

Fuente: Albrieu R. et al. (2015). Argentina: una estrategia de desarrollo para el siglo XXI, edición Turmalina, p. 54.

Gráfica 5. Brasil: Tasa de cambio real efectivo, febrero de 1999 a julio de 2014, vela la crisis (Año $2000=$ Base 100)

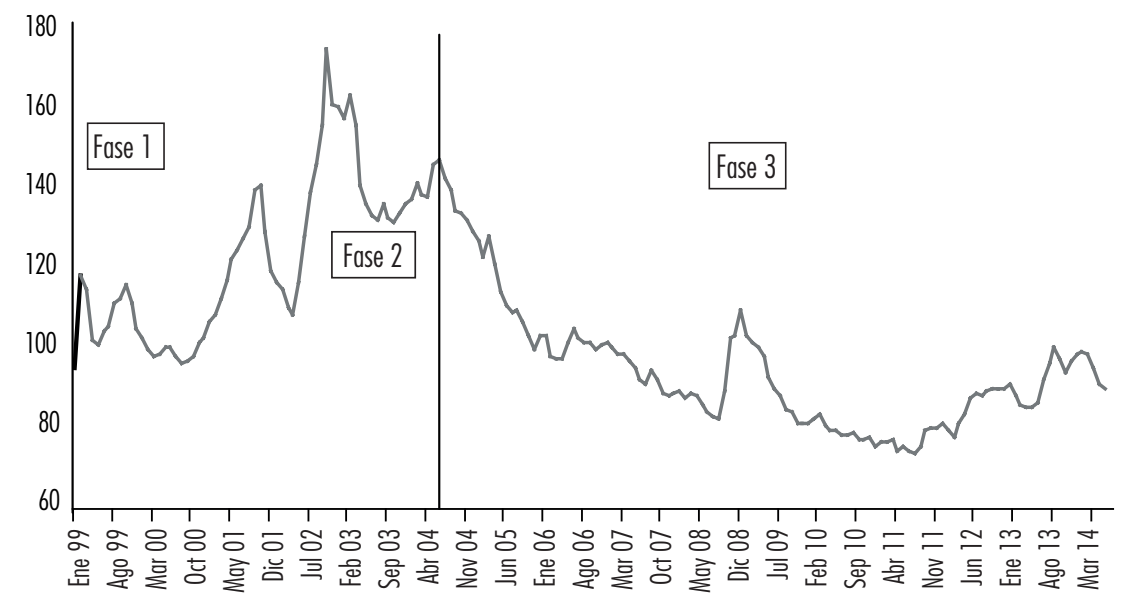

Fuente: Banco Central de Brasil. 
la moneda nacional. Los tipos de cambio son expresados en términos reales con el objetivo de tener en cuenta diferenciales de inflación con Estados Unidos.

La apreciación de la moneda nacional respecto al dólar es un obstáculo a una mayor competitividad, no solamente porque son menos costosos los productos importados, y a la inversa, más caros los productos exportados, sino también porque desalienta la inversión en el sector de mayor riesgo, permite la especulación inmobiliaria y de manera general se reorienta la inversión en los sectores no riesgosos, y en el sector productor de materias primas. Al final la tasa de inversión apenas si crece y dificulta mantener una tasa de crecimiento del PIB en un nivel elevado (véase cuadro 2).

La debilidad relativa de la tasa de inversión en el ramo de la industria, no impulsa un aumento de la productividad del trabajo, ya que, el esfuerzo en términos de investigación y desarrollo no es coherente ( $1 \%$ del PIB), sobre todo si se le compara con lo hecho por Corea del Sur (más de 4\%). La productividad del trabajo en el sector industrial, en efecto, crece relativamente poco: $17 \%$ entre 2004 y 2015 . El salario real promedio aumenta el $34 \%$ en el mismo periodo, más que la productividad (véase cuadro 3 ).

Para una base 100 en 2004, el índice de precios de las importaciones de bienes de consumo no duraderos es de 150 en 2014, en vísperas de la crisis y de la fuerte depreciación del real, el de los bienes de consumo duraderos es 113 , el de los bienes intermedios es 120 , el de los bienes de equipo es 100 en la misma fecha. Aún, para una base 100 en 2004, el índice del costo unitario del trabajo en la industria de transformación sobrepasa ampliamente a los bienes importados para situarse en 221 (CEMEC, 2015). Los tres componentes del costo unitario del trabajo evolucionan desfavorablemente (apreciación de la moneda que se refleja en los precios importados, alza de los salarios que sobrepasa un crecimiento muy débil de la productividad), la competitividad del país declina fuertemente. Es más baja que la de los bienes sofisticados, salvo en algunas ramas como la aeronáutica. Aun cuando comprendemos, que la balanza comercial de productos de la industria de transformación se haya vuelto negativa en 2008, en realidad, posteriormente se volvió más negativa hasta 2014 (más de 25 mil millones de dólares de déficit). ${ }^{6}$ El aumento de la

No fue, sino a partir del segundo semestre que el déficit se redujo, después de la máxima depreciación de la moneda (de octubre de 2014 a octubre de 2015, la depreciación de la moneda es de $47.24 \%$ según el Banco Central, y en términos reales es de 37\%), las exportaciones cayeron menos que las importaciones. Fue hasta finales de 2015 que las exportaciones empezaron a aumentar, al paso de los meses, cada vez con mayor fuerza. El saldo negativo de la balanza comercial se hace cada vez más positivo a pesar de la caída de los precios de las materias primas. Tan sólo para el mes de marzo de 2016 fue de 4.43 mil millones de dólares. 
Cuadro 2. Brasil: Tasa de inversión y de ahorro bruto, tasa de crecimiento del PIB, 2010-2015

\begin{tabular}{lrrrrrr} 
& 2010 & 2011 & 2012 & 2013 & 2014 & 2015 \\
\hline Tasa de Inversión & 20.5 & 20.6 & 20.7 & 20.9 & 20.2 & 18.2 \\
Tasa de Ahorro & 17.9 & 18.5 & 18 & 18.4 & 16.2 & 14.4 \\
Tasa de Crecimiento del PIB & 7.5 & 3.9 & 1.9 & 3.0 & 0.1 & -3.8 \\
\hline
\end{tabular}

Fuente: IBGE.

Cuadro 3. Productividad y salarios reales en la industria de la transformación en moneda nacional (Año base 2004=100)

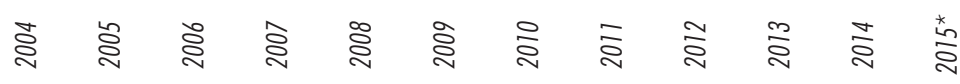

$\begin{array}{lllllllllllll}\text { Productividad } & 100.0 & 102.0 & 103.5 & 107.5 & 107.5 & 105.5 & 115.0 & 115.0 & 112.5 & 117.0 & 117.0 & 116.0\end{array}$

$\begin{array}{lllllllllllll}\text { Salario } & 100.0 & 102.5 & 102.5 & 106.0 & 110.0 & 115.0 & 118.5 & 122.5 & 128.5 & 132.0 & 134.0 & 133.0\end{array}$

*janvier- aout 2015.

Fuente: CEMEC, note 08/2015.

demanda, en respuesta al aumento de los salarios, no generó un aumento de la producción industrial, sino un aumento de las importaciones.

La producción industrial se estanca y se hunde con la crisis. Para un índice promedio de 100 en 2002 (dado el desaliento), alcanza un pico de 105.5 en junio de 2013, luego 99 en noviembre de 2014, y 85 en enero de 2016 y 83 en febrero de 2016, a pesar de la revivificación de las exportaciones la industria continua su de decadencia (carta IEDI, $n^{\circ} 726$ ). Si no se tiene en cuenta la crisis de 2015-2016, en 11 años la industria de transformación no creció. Pasado un cierto estado de desarrollo, es habitual constatar una caída relativa del sector industrial en el PIB a favor del sector servicios, sin que por eso hubiera necesariamente desindustrialización.

El término desindustrialización es generalmente reservado para una disminución absoluta del valor agregado industrial, y/o una reducción relativa del peso de la industria nacional en la industria mundial en las economías abiertas. En América Latina, este fenómeno tiende a ocurrir mucho antes que en los países avanzados debido a la clasificación "temprana” utilizada cuando 
el ingreso per cápita, a inicios del proceso de desindustrialización corresponde a la mitad del ingreso per cápita obtenido en los países avanzados al momento que da principio dicha condición.

La participación de la industria de transformación brasileña en la industria de transformación mundial (en valor agregado) es de $1.8 \%$ en 2005 , de $1.7 \%$ en 2011 despúes de haber alcanzado $2.8 \%$ en 1980, según la información bancaria para 2013 de la UNCTAD. De acuerdo con la misma fuente, en China esta proporción fue del $9.9 \%$ en 2005 y $16.9 \%$ en 2011 . Por lo tanto, fue relativamente más baja en Brasil, mientras que aumentó considerablemente en China. Las exportaciones brasileńas de productos manufacturados disminuyeron en términos relativos, pasaron del 53\% del valor de las exportaciones en 2005 a 35\% en 2012, a favor de las exportaciones de materias primas agrícolas y mineras, y no es hasta febrero de 2016 que crecen nuevamente debido a la fuerte devaluación y el colapso de los precios de las materias primas como lo hemos visto.

\section{CONCLUSIONES Y REFLEXIONES PARA CAMBIAR EL FUTURO}

¿Quién cometió el error, a quién culpar? ¿China, o los gobiernos brasileños que se sucedieron? La desindustrialización observada es una reminiscencia de una "enfermedad holandesa". Sabemos que no es ineluctable. La vía para salir de la trampa rentista es estrecha, pero existe. Los gobiernos brasileños, prefirieron no pedir prestado, convencidos de que los precios de las materias primas continuarían aumentando, y eso les permitiría, sin hacer reformas estructurales como por ejemplo una reforma fiscal a profundidad, evitar conflictos importantes para aplicar una política orientada a reducir la pobreza, dejando al $1 \%$ de los más ricos hacerse todavía más ricos. La reprimarización consolidó los comportamientos rentistas presentes desde hace mucho tiempo, herencia del pasado.

La reanudación del crecimiento a través de una reindustrialización es posible. El auge de las materias primas parece haber terminado. Aun así, esta actividad continúa proporcionando muchas divisas a Brasil. Éstas podrían servir de trampolín para una inserción más positiva en la división internacional del trabajo. Para quienes sueñan con la exportación de productos agrícolas, incorporar más valor agregado y adecuarse a las tendencias de consumo en China como lo pronostica el Instituto de Pesquisa Económica Aplicada (IPEA). De manera más general, la reindustrialización requiere que se cumplan cinco condiciones: 1) un incremento importante de la productividad del trabajo, 
reduciendo el costo unitario de la mano de obra; 2) cuestionar la lógica de las economías rentistas que limita al mínimo la tasa de inversión, a una porción congruente con una política industrial del Estado para ayudar a los sectores de punta $-\mathrm{y}$ no a toda la industria-, que pueden necesitar temporalmente de medidas proteccionistas; 3) una política activa orientada a neutralizar la apreciación de la moneda; 4) una reforma fiscal que permita disminuir las desigualdades de ingresos y mejorar el poder adquisitivo de las clases pobres vulnerables, y de los estratos medios bajos (emergentes), y financiar los servicios públicos de calidad (educación, salud...), y 5) una política que facilite el acceso al crédito para pobres y estratos sociales vulnerables. Que una de estas condiciones no se cumpla, hace más aleatorio el éxito de una política de crecimiento impulsado por la dinámica del mercado interno.

\section{BIBLIOGRAFÍA}

Banco Mundial (2016), Commodity Market Outlook, Quaterly Report, January.

BU, Global Economic Governance Initiative (2016), "China Latin America Economic Bulletin”, disponible en <https://www.bu.edu/pardeeschool/ files/2016/04/China-LAC.Bulletin_2016.pdf>

Cardim de Carvalho, Fernando (2016), "The Narrow Path for Brazil", Levy Economics Institute for Bard College, Policy Note, Estados-Unidos, pp. 1-10.

China-Latin America Economic Bulletin (2016), "Global Economic Governance Initiative", Discussion Paper.

Costa Pinto, Eduardo y Reinaldo Gonçalves (2015), "Globalização e poder efetivo: transformações globais sob efeito da ascensão chinesa", Economia e Sociedade, Campinas, Unicamp. IE. v. 24, n. 2 (54), Brasil, pp. 449-479.

Dussel Peters, Enrique (2016), "A relaçao de comercio e de investimentos de América Latina com a China (2000-2012), uma nova forma de dependencia”, Revista Tempo du Mundo, Brasil, IPEA, vol. 2, núm.1, pp. 123-142.

Dussel Peters, Enrique y Samuel Ortiz Velásquez (2016), Monitor de la OFDI de China en México, año 1, núm. 1.

Foster, Magdalena (2015), "China rebalancing: Blessing and Curse for Latin America Research Briefing", Emerging Markets, Deutsche Bank Research, pp. 1-6.

Hiratuka Célio y Fernando Sarti (2016), "Relaçóes econômicas entre Brasil e China: análise dos fluxos de comércio e investimento direto estrangeiro", Revista Tempo du Mundo, Brasil, IPEA, vol. 2, núm. 1, pp. 83-98. 
Kevin, P. Gallagher (2016), The China Triangle: Latin America's China Boom and the Fate of the Washington Consensus, Oxford, Oxford University Press.

Macedo Cintra, Marcos Antonio y Eduardo Costa Pinto (2013), "América Latina y China en el siglo xxi: complementariedades y rivalidades", Revista Vozes en el Fenix, Brasil, pp. 86-95.

Nassif A, Feijo, C., Araujo, E. (2015), "Structural Change and Economic Development: Is Brazil catching up or falling behind?", Cambridge Journal of Economics, 39, pp. 1307-1332.

OCDE/Cepal/CAF (2015), "Perspectivas económicas de América Latina 2016: Hacia una nueva asociación con China”, oecd Publishing, Paris.

Salama, Pierre (2012a), "China-Brasil: industrialización y desindustrialización temprana", Cuadernos de Economia, 31 (56), Colombia, pp. 223-252. (2012b), "Globalización comercial: desindustrialización prematura en América Latina e industrialización en Asia”, Comercio Exterior, vol. 62, núm. 6, México, pp. 34-44.

(2012c), "Una globalización comercial acompañada de una nueva distribución cartográfica”, Economía Institucional, vol. 14, núm. 27, Colombia, pp. 57-80.

(2012d), Les économies émergentes latino-américaines, entre cigales et fourmis, Armand Colin, Collection U, publication en cours en espagnol (2016) con postfacio, Universidad de Guadalajara.

(2015), "China-Brasil, una comparación instructiva”, Foro Internacional, vol. Lv, núm. 220, México, pp. 515-624.

Trebat, Nicholas y Carlos Aguiar de Medeiros (2014), "Military Modernization in Chinese Technical Progress and Industrial Innovation", Review of Political Economy, Inglaterra, vol. 26, Issue 2, pp. 303-324.

Vieira Pedro, Abel, Antônio Marcio Buainain y Eliana Valeria Covolan Figueiredo, (2016), "O Brasil alimentará a China ou a China engolirá o Brasil?”, Revista Tempo du Mundo, Brasil, IPEA, vol. 2, núm. 1, pp. 51-82.

\section{INSTITUCIONES INTERNACIONALES}

OCDE: www.oecd.org

Cepal: www.eclac.org

BID: www.iadb.org

ADB: www.adb.org 